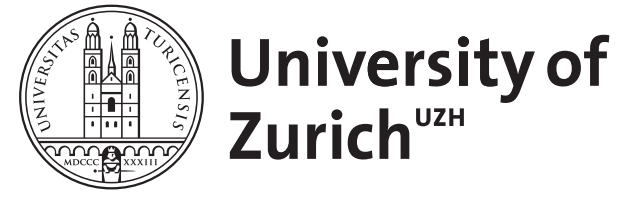

\title{
Psychotherapie für alle?
}

\author{
Schnyder, U ; McShine, R M ; Kurmann, J ; Rufer, M
}

\begin{abstract}
Background: The effectiveness of psychotherapy for the treatment of most mental disorders is empirically very convincingly documented; however, there are not enough therapists by far available globally to enable all people suffering from mental disorders to be adequately provided with psychotherapy. Aims and methods: Considerations are made regarding which illnesses, disorders and problems in general should be treated by means of psychotherapy, who should best conduct the treatment and in which way the dissemination of evidence-based psychotherapeutic approaches could be improved in spite of scarce resources. Results: The more severely pronounced a health problem is, the greater is normally the therapeutic benefit of a given intervention. This applies to psychotherapy as well; however, to date severely ill people in particular are often not treated with effective psychotherapeutic interventions. One of the reasons is that there are only few validated treatment protocols for multimorbid patients with both mental and physical illnesses. Another reason is that treatment of such patients requires specific medical knowledge as well as other special skills and experiences that not all psychotherapists have at their command. Conclusion: The indications for psychotherapy should always be made with a sense of proportion, taking into consideration the currently available scientific evidence and clinical experience. In the future, the training of psychotherapists, scientific investigations of psychotherapies and clinical service provision should increasingly concentrate on patients with severe mental disorders and/or with psychological and physical comorbidities.
\end{abstract}

DOI: https://doi.org/10.1007/s00115-013-3976-4

Posted at the Zurich Open Repository and Archive, University of Zurich ZORA URL: https://doi.org/10.5167/uzh-103868

Journal Article

Published Version

Originally published at:

Schnyder, U; McShine, R M; Kurmann, J; Rufer, M (2014). Psychotherapie für alle? Der Nervenarzt, 85(12):1529-1535.

DOI: https://doi.org/10.1007/s00115-013-3976-4 
Nervenarzt 2014 $\cdot 85: 1529-1535$

DOI 10.1007/s00115-013-3976-4

Online publiziert: 5. Dezember 2014

(c) Springer-Verlag Berlin Heidelberg 2014
U. Schnyder ${ }^{1}$ - R.M. McShine ${ }^{2}$. J. Kurmann ${ }^{3}$ - M. Rufer ${ }^{1}$

${ }^{1}$ Klinik für Psychiatrie und Psychotherapie, Universitätsspital Zürich

${ }^{2}$ Integrierte Psychiatrie Winterthur-Zürcher Unterland, Winterthur

${ }^{3}$ Luzerner Psychiatrie, St. Urban

\section{Psychotherapie für alle? Zur Indikation für psychotherapeutische
Behandlungen}

\begin{abstract}
Die Psychotherapie zählt zu den wirksamsten Verfahren in der Medizin. Wir verfügen heute über eine ganze Palette von „empirically supported therapies" für fast alle psychiatrischen Störungsbilder und Behandlungssettings. Die verfügbaren Ressourcen reichen aber bei weitem nicht aus, um alle Menschen mit psychischen Erkrankungen psychotherapeutisch behandeln zu können. Auch wenn Psychotherapie im Vergleich zu anderen medizinischen Leistungen sehr günstig ist, werden ökonomische Überlegungen den Zugang zur Psychotherapie als Krankenkassenleistung voraussichtlich zunehmend limitieren.
\end{abstract}

Gemäß dem integrativen Psychotherapie-Lehrbuch von Wolfgang Senf und Michael Broda [21] ist Psychotherapie wissenschaftlich fundiertes, professionelles Handeln im Rahmen und nach den Regeln des öffentlichen Gesundheitswesens. Eine derart positionierte Psychotherapie gründet auf wissenschaftlich nachvollziehbaren und empirisch überprüften Krankheits-, Heilungs- und Behandlungstheorien. Sie setzt theoretisch abgeleitete und empirisch abgesicherte Verfahren zur zielgerichteten Veränderung im Erleben und Verhalten von Patienten ein und bezweckt damit die Behandlung psychisch bedingter oder mitbedingter Krankheiten, Störungen und Beschwerden. Wir sprechen hier also von einer evidenzbasierten Psychotherapie. Aber lassen sich die Forderungen einer „evidence based medicine" auf den Bereich der Psychotherapie übertragen, im Sinne einer „evidence based psychotherapy“ [13]?

Im Prinzip ja: Die Wirksamkeit der Psychotherapie zur Behandlung der meisten psychischen Störungen ist heute empirisch gut belegt. Aus Sicht der ,evidencebased medicine" kann Psychotherapie als einer der wirksamsten Therapieansätze in der Medizin gelten [4, 6, 11, 12]. Insbesondere im Langzeitverlauf ist sie der Psychopharmakotherapie bei den meisten psychischen Erkrankungen überlegen. Kognitiv-verhaltenstherapeutische Ansätze wie Expositions-Reaktions-Management oder kognitive Umstrukturierung, aber auch andere, psychodynamisch oder systemisch orientierte Interventionen verfügen heute über einen robusten Wirksamkeitsnachweis, sodass wir über eine ganze Palette von „empirically supported therapies" [1] für unterschiedliche Patientengruppen und Behandlungssettings verfügen [7].

\section{》) Nicht nur Ärzte beherrschen Psychotherapie}

Psychotherapie zur Behandlung psychischer Störungen wird oft von Fachärzten (für Psychiatrie und Psychotherapie, für psychotherapeutische Medizin, für psychosomatische Medizin und Psychotherapie etc.) in einem medizinischen Behandlungsrahmen durchgeführt [19], in psychiatrisch-psychotherapeutischen stationären und ambulanten Einrichtungen wie auch in ärztlichen Privatpraxen. Einige
Spezifika werden unter der Bezeichnung „ärztliche Psychotherapie“ diskutiert [2]. Psychotherapie wird jedoch sehr häufig, sei es in medizinischen oder in anderen Settings, von nichtärztlichen Berufsgruppen angeboten, z. B. von klinischen Psychologen, Sozialarbeitern, Seelsorgern, Pflegefachleuten. In einzelnen Ländern wie z. B. Österreich wurden zudem in den letzten Jahren die gesetzlichen Grundlagen geschaffen, die es ermöglichen, Psychotherapie als Beruf zu studieren, ohne vorgängig Medizin, Psychologie oder einen anderen Grundberuf gelernt zu haben. Psychotherapie wird also schon lange nicht mehr nur unter medizinischen Rahmenbedingungen eingesetzt, und es sind auch längst nicht mehr ausschließlich Ärzte, welche Psychotherapie beherrschen und anwenden.

Damit ist die Frage, wer denn von wem psychotherapeutisch behandelt werden soll, etwas komplexer und deshalb auch schwieriger zu beantworten als beispielsweise in der Chirurgie. Die Indikation für einen chirurgischen Eingriff wird durch einen Facharzt für Chirurgie gestellt, und die Operation wird in aller Regel durch einen Chirurgen durchgeführt. Zwar sind die Grenzen auch hier nicht mehr so eindeutig und klar wie noch vor einigen Jahrzehnten: Die Kardiologie hat sich beispielsweise zu einer interventionellen Disziplin gewandelt, sodass heute Herzchirurgen mit Kardiologen gemeinsam in sog. Hybrid-Operationssälen arbeiten; auch die Neuroradiologen stehen nicht mehr "nur" im radiologischen Befundraum, sondern sie verstehen sich als Operateu- 
re, wenn sie z. B. bildgebungsunterstützt intrakranielle vaskuläre Eingriffe vornehmen. Im Feld der Psychotherapie sind die Grenzen aber dennoch viel weniger scharf. Zwar ist in den meisten Ländern einigermaßen klar definiert, wer sich Psychotherapeut nennen darf. Die Unschärfe beginnt aber bereits bei der Frage, welche Krankheiten, Störungen oder Probleme generell psychotherapeutisch behandelt werden sollen. Dann: Welche spezifischen Interventionen sind bei welchen Situationen indiziert? Wer soll Psychotherapien durchführen dürfen? Gibt es Problemfelder, die nur von speziell ausgebildeten Psychotherapeuten behandelt werden dürfen? Für was sollte Psychotherapie nicht angeboten werden?

\section{Psychotherapie für alle?}

Das Feld der Psychotherapie ist im deutschsprachigen Raum über die $\mathrm{Zu}$ lassung zur finanziellen Abgeltung über Leistungen zulasten der Krankenkassen reguliert. Dennoch spielt auch der freie Markt zu einem gewissen Grad eine Rolle. Das hat zur Folge, dass insbesondere in den psychotherapeutischen Praxen heute viele Patienten behandelt werden, die eher zu den leicht kranken Menschen gezählt werden können. Aus einer globalen „Public-mental-health“-Perspektive scheint es aber durchaus sinnvoller, Psychotherapie primär den Menschen mit schweren psychischen Erkrankungen zugutekommen zu lassen. Gerade die psychischen Erkrankungen mit den höchsten Lebenszeitprävalenzen, wie z. B. Depressionen, Angsterkrankungen und Substanzabhängigkeiten, vielleicht am ehesten noch mit Ausnahme der organischen psychischen Störungen (Demenzen, Delirien), sollten primär psychotherapeutisch behandelt werden. Wir wissen beispielsweise für die Depression, dass selbst in einem hochentwickelten Land wie Deutschland nur ein sehr geringer Anteil, d. h. weniger als 5\% der Betroffenen, tatsächlich eine umfassende professionelle und somit auch psychotherapeutische Behandlung erhält. In vielen anderen Ländern sieht die Situation noch viel schlimmer aus. Mit anderen Worten: Die weltweit verfügbaren personellen Ressourcen reichen heute und bis auf absehbare Zeit bei weitem

Nervenarzt 2014 · 85:1529-1535 DOI 10.1007/s00115-013-3976-4

(c) Springer-Verlag Berlin Heidelberg 2014

\section{U. Schnyder · R.M. McShine · J. Kurmann · M. Rufer \\ Psychotherapie für alle? Zur Indikation für psychotherapeutische Behandlungen}

\section{Zusammenfassung}

Hintergrund. Die Wirksamkeit der Psychotherapie zur Behandlung der meisten psychischen Störungen ist empirisch sehr überzeugend belegt. Es gibt aber weltweit bei weitem nicht genügend Therapeuten, um alle Menschen mit psychischen Erkrankungen angemessen psychotherapeutisch behandeln zu können.

Fragestellung und Methodik. Es werden Überlegungen zu der Frage dargestellt, welche Krankheiten, Störungen oder Probleme generell psychotherapeutisch behandelt werden sollen, wer diese Behandlungen am besten durchführt und wie die Dissemination evidenzbasierter Ansätze trotz knapper Ressourcen verbessert werden kann.

Ergebnisse. Je schwerer eine Gesundheitsstörung ausgeprägt ist, umso höher ist in aller Regel auch der therapeutische Nutzen einer Intervention. Das gilt auch für die Psychotherapie. Aber gerade die schwer kranken Menschen werden heute oft nicht mit wirksamen psychotherapeutischen Interventionen behandelt. Ein Grund hierfür ist, dass es wenig validierte Therapiekonzepte für multi- morbide Patienten mit psychischen und somatischen Erkrankungen gibt. Ein weiterer Grund besteht darin, dass deren Behandlung ein spezifisches medizinisches Wissen sowie weitere spezielle Fähigkeiten und Erfahrungen erfordert, die bei weitem nicht alle Psychotherapeuten besitzen.

Schlussfolgerungen. Die Indikation zur Psychotherapie soll immer sorgsam, mit Augenmaß, unter Berücksichtigung der verfügbaren wissenschaftlichen Evidenz und klinischer Erfahrungswerte gestellt werden. Zukünftig sollten sich die Ausbildung von Psychotherapeuten, die wissenschaftlichen Untersuchungen von Psychotherapien und die klinischen Angebote verstärkt auf Patienten mit schweren psychischen Störungen und/oder mit psychischen und somatischen Komorbiditäten konzentrieren.

\section{Schlüsselwörter}

Psychotherapie - Lifestyle .

Evidenzbasierte Medizin - Therapeutischer Nutzen · Multimorbidität

\section{Psychotherapy for everyone? On the indications for psychotherapeutic treatment}

\section{Summary}

Background. The effectiveness of psychotherapy for the treatment of most mental disorders is empirically very convincingly documented; however, there are not enough therapists by far available globally to enable all people suffering from mental disorders to be adequately provided with psychotherapy. Aims and methods. Considerations are made regarding which illnesses, disorders and problems in general should be treated by means of psychotherapy, who should best conduct the treatment and in which way the dissemination of evidence-based psychotherapeutic approaches could be improved in spite of scarce resources.

Results. The more severely pronounced a health problem is, the greater is normally the therapeutic benefit of a given intervention. This applies to psychotherapy as well; however, to date severely ill people in particular are often not treated with effective psychotherapeutic interventions. One of the reasons is that there are only few validated treatment protocols for multimorbid patients with both mental and physical illnesses. Another reason is that treatment of such patients requires specific medical knowledge as well as other special skills and experiences that not all psychotherapists have at their command. Conclusion. The indications for psychotherapy should always be made with a sense of proportion, taking into consideration the currently available scientific evidence and clinical experience. In the future, the training of psychotherapists, scientific investigations of psychotherapies and clinical service provision should increasingly concentrate on patients with severe mental disorders and/or with psychological and physical comorbidities.

\section{Keywords}

Psychotherapy · Lifestyle - Evidence-based medicine - Therapeutic benefits .

Multimorbidity 
Hier steht eine Anzeige.

黛 Springer 
nicht aus, um auch nur die Menschen mit den schwersten psychischen Erkrankungen angemessen psychotherapeutisch behandeln zu können.

Das wäre an sich ein starkes Argument dafür, Psychotherapie wirklich nur einem Segment von sehr schwer kranken Menschen zukommen zu lassen. Man könnte mit guten Gründen sogar noch weiter gehen und dafür plädieren, dass wir uns hauptsächlich auf multimorbide Menschen, also auf Patienten mit vielfältigen psychischen und somatischen Komorbiditäten konzentrieren sollten. Zumindest im deutschsprachigen Raum, für den diese Arbeit geschrieben wurde, sind wir jedoch mit einer anderen Realität konfrontiert: Gerade schwer kranke und multimorbide Menschen werden sehr oft nicht mit wirksamen psychotherapeutischen Interventionen behandelt.

\section{》) Psychotherapie \\ sollte vermehrt den sehr schwer kranken Menschen angeboten werden}

Dies hat sicher viele Gründe. Einer davon ist nicht zuletzt, dass es bis heute noch kaum validierte Therapiekonzepte für multimorbide Menschen mit psychischen und somatischen Erkrankungen gibt. Ein weiterer Grund besteht in der oben erwähnten Vielfalt der Berufsgruppen, die Psychotherapie anbieten. Die Behandlung von Menschen mit schweren somatischen und psychischen Erkrankungen erfordert ein spezifisches Wissen, spezielle Fähigkeiten und Erfahrungen, die Ärzte während ihrer gesamten, über 10 Jahre dauernden Aus- und Weiterbildung fundiert lernen und praktizieren. Psychotherapeuten anderer Berufsgruppen haben hier häufig weniger Erfahrung und bieten solchen Patienten (durchaus zu Recht, aufgrund ihrer nicht ausreichenden diesbezüglichen Kompetenz) keine Psychotherapie an, beenden eine solche früh oder behandeln nur unspezifischsupportiv. Beispiele hierfür gibt es viele, wir nennen hier nur einige wenige: Panikstörungen bei Zustand nach Herzinfarkt und Herzinsuffizienz; schwere Depressionen bei AIDS-Erkrankung und Interferontherapie wegen Hepatitis C; gemischte epileptische und dissoziative Krampfan- fälle; posttraumatische Belastungsstörungen (PTSD) und invalidisierende somatisch und psychisch verursachte Schmerzen nach schwerem Unfall oder bei Folteropfern. Ähnliches gilt für Patienten, die neben einer psychotherapeutisch sehr gut behandelbaren Erkrankung (z. B. Angstoder Zwangsstörung, Depression, PTSD) eine weitere, schwere psychiatrische Erkrankung aufweisen, z. B. eine Schizophrenie oder bipolare affektive Störung.

Je schwerer eine Gesundheitsstörung ausgeprägt ist, umso höher ist in aller Regel auch der therapeutische Nutzen einer Intervention. Das gilt gleichermaßen für die Pharmakotherapie wie auch für die Psychotherapie, und insbesondere für akute psychische Erkrankungen. Dauert die Erkrankung - wie bei vielen psychischen Störungen - länger an, wird der mögliche therapeutische Gewinn durch Chronifizierungsprozesse und die damit verbundene Entmutigung auf Seiten der Patienten wie auch der Therapeuten teilweise reduziert. Deshalb muss in der Psychiatrie die Wirksamkeit einer Heilmethode daran gemessen werden, inwiefern sie im Langzeitverlauf über mehrere Jahre zu einer klinisch relevanten Besserung führt. Genau hier liegt die Stärke der Psychotherapie, lässt sich doch häufig - im Unterschied zur alleinigen Pharmakotherapie - im Langzeit-Follow-up nach Abschluss einer psychotherapeutischen Behandlung eine Stabilität des Therapieerfolges oder sogar eine weitere Verbesserung im Hinblick auf Symptomatik und Lebensqualität nachweisen [5].

$\mathrm{Zu}$ bedenken ist allerdings, dass Psychotherapie bei leichteren psychischen Störungen nicht nur gute Chancen auf Besserung oder Heilung bietet, sondern auch verhindert, dass die Erkrankung fortschreitet, chronifiziert und zu Folgeerkrankungen führt. Angesichts dieser Überlegungen plädieren wir dafür, dass grundsätzlich alle Menschen mit einer psychischen Störung, unabhängig vom Schweregrad der Erkrankung, bei entsprechender Indikationsstellung die Möglichkeit einer psychotherapeutischen Behandlung bekommen sollten. Zukünftig sollte Psychotherapie aber verstärkt auch den Menschen zugutekommen, die eine wirksame Therapie am dringendsten brauchen, d. h. Patienten mit schweren chro- nischen psychischen Störungen, Patienten mit komplexen, komorbiden Störungsbildern und Patienten mit zusätzlichen gesundheitlichen Problemen, d. h. mit komorbiden somatischen Erkrankungen.

\section{Dissemination evidenzbasierter Psychotherapie}

Weiter würden wir dafür plädieren, dass die erwähnten Patienten nicht irgendeine, sondern eine möglichst wirksame Psychotherapie erhalten sollten. Es gibt im deutschsprachigen Raum eine Vielzahl gut ausgebildeter und klinisch erfahrener Psychotherapeuten in entsprechenden Institutionen und in der freien Praxis. Für die häufigsten psychischen Störungen verfügen wir heute über sehr wirksame Behandlungsmöglichkeiten, und unsere Patienten haben meistens auch eine Krankenversicherung, die den größten Teil der Behandlungskosten decken würde. Trotzdem werden die meisten psychisch kranken Menschen entweder überhaupt nicht professionell behandelt oder sie erhalten eine nur suboptimal wirksame Therapie [14]. Woran liegt das? Zum einen haben viele Psychotherapeuten wenig Erfahrung in empirisch abgesicherten Therapieverfahren, auch weil sie nicht gerne mit Therapiemanualen arbeiten. Auch heute noch wird, entgegen der empirischen Erkenntnisse, zum Teil befürchtet, dass die Befolgung manualisierter Therapieschritte die kreative Entfaltung des Therapeuten einschränkt und deshalb den Patienten nicht hinreichend gerecht wird. Auf der anderen Seite verlangen aber Patienten und auch Kostenträger zunehmend den Einsatz evidenzbasierter Verfahren. Der Siegeszug der „evidence based medicine“ und der „informierte Patient", den wir uns ja wünschen, lassen uns keine Wahl: Wir werden uns in Zukunft vermehrt der Frage stellen müssen, inwiefern die Wirksamkeit unserer therapeutischen Interventionen empirisch nachgewiesen ist. Es ist zu hoffen und zu erwarten, dass sich dadurch die empirisch abgesicherten psychotherapeutischen Verfahren mit der Zeit besser durchsetzen werden.

$\mathrm{Zu}$ einer besseren Dissemination evidenzbasierter Psychotherapieansätze wird eine Entwicklung beitragen, welche moderne Kommunikationstechnologien für 
Diagnostik und Therapie nutzt: Die sog. „telehealth" oder „telemedicine“ [16]. Per Videokonferenzen wird klinische Diagnostik oder Supervision betrieben, therapeutische Interventionen werden webbasiert oder via Smartphone appliziert. Im Bereich der Psychiatrie und Psychotherapie wurden im Rahmen solcher „telemental health approaches “ für ganz unterschiedliche Störungsbilder und Problemfelder internetbasierte Interventionsprotokolle entwickelt [15]. Heute gibt es bereits eine Vielzahl webbasierter Selbsthilfeangebote, aber auch Psychotherapien im engeren Sinne. Die Angebote richten sich häufig an Menschen mit leichten bis mittelschweren Erkrankungen, aber zumindest zum Teil auch an solche mit komplexen Störungen, beispielsweise Depressionen bei Multipler Sklerose. Sie scheinen gut zu funktionieren und von vielen, vorderhand noch hauptsächlich jüngeren $\mathrm{Pa}$ tienten, rege genutzt zu werden. Die meisten internetbasierten Therapieprotokolle bringen kognitiv-verhaltenstherapeutische Elemente (Psychoedukation, Selbstkonfrontation bzw. Exposition, kognitive Umstrukturierung) zur Anwendung. Die überwiegende Mehrheit der behandelten Patienten gibt an, dass sie den Kontakt mit ihrem Therapeuten als „persönlich“ erleben, dass sie das Internet als Kommunikationsmedium angenehm finden und dass sie keine Probleme damit haben, ihren Therapeuten nie direkt zu Gesicht zu bekommen [9]. In Metaanalysen wird über signifikante, allerdings eher schwach bis mittelstark ausgeprägte Therapieeffekte berichtet [22].

\section{》) "Telemental health" ist ein wertvolles ergänzendes Behandlungsangebot}

In den meisten Ländern werden internetbasierte Therapien in der Regel (noch) nicht von den Kostenträgern übernommen. Trotzdem stellen sie bereits heute ein wertvolles ergänzendes Behandlungsangebot dar, z. B. für Menschen, die in medizinisch und therapeutisch stark unterversorgten Gegenden dieser Welt leben oder körperlich behindert oder anderweitig häuslich gebunden sind. Sie können auch eine Alternative für Menschen darstellen, die Angst vor einem direkten therapeutischen Kontakt bzw. einer möglichen Stigmatisierung haben (z. B. Menschen in Helferberufen, Polizei, Feuerwehr etc.). „Telemental-health“-Ansätze öffnen somit das Feld der Psychotherapie für ein wesentlich erweitertes Publikum. Sie sind zweifellos nicht ohne Risiken, beispielsweise hinsichtlich Datenschutz, stellen aber vor allem aus einer globalen „Public-mental-health“-Perspektive und auch in ökonomischer Hinsicht eine sehr interessante Option und Ergänzung zur klassischen „Face-to-face“-Psychotherapie dar.

\section{Psychotherapie durch wen?}

Wer soll Psychotherapien durchführen dürfen? Wir fragen hier nicht aus einer standespolitischen, sondern aus einer fachlichen Perspektive. Es ist, wie oben beschrieben, längstens eine Realität, dass Psychotherapie von Fachpersonen mit sehr unterschiedlichen beruflichen Grundausbildungen ausgeübt wird. Wir sollten uns deshalb überlegen, welches die fachlichen Voraussetzungen dafür sind, Psychotherapie als Beruf ausüben zu können.

Im Jahr 1998 schrieb der Nobelpreisträger Eric Kandel:

Insofar as psychotherapy or counseling is effective and produces long-term changes in behavior, it presumably does so through learning, by producing changes in gene expression that alter the strength of synaptic connections and structural changes that alter the anatomical pattern of interconnections between nerve cells of the brain. As the resolution of brain imaging increases, it should eventually permit quantitative evaluation of the outcome of psychotherapy. [8]

Mittlerweile ist die „Neuropsychotherapie“ [3] eine Realität geworden. Unabhängig von seinem beruflichen Hintergrund muss ein guter Psychotherapeut heute nicht nur über gründliche Kenntnisse in Entwicklungspsychologie, Psychopathologie und den wichtigsten psychologischen Therapiekonzepten und Behandlungstheorien verfügen sowie eine Reihe psychotherapeutischer Techniken und Fertigkeiten beherrschen. Er muss sich ebenso in den Bereichen der Neurobiologie und Psychophysiologie, der Kognitionswissenschaften sowie der Sozial- und Kulturpsychologie auskennen [20], nicht zuletzt um, angesichts der zunehmenden Globalisierung unserer Gesellschaft, auch ein „kultur-sensitiver“ Psychotherapeut zu sein [23], der bei jeder therapeutischen Interaktion die kulturelle Dimension mitberücksichtigt.

Mit anderen Worten, Psychotherapie ist eine sowohl intellektuell als auch emotional und „menschlich“ anspruchsvolle Tätigkeit. Für uns folgt daraus, dass Psychotherapie von akademisch ausgebildeten, emotional belastbaren, beziehungsfähigen und kognitiv flexiblen, klinisch versierten Fachpersonen ausgeübt werden sollte, die idealerweise über eine gewisse persönliche Reife, Lebenserfahrung und Selbstreflexionsfähigkeit verfügen. Wir sind uns dabei bewusst, dass sich Begriffe wie emotionale Belastbarkeit, Beziehungsfähigkeit, Reife und Lebenserfahrung nicht leicht operationalisieren lassen. Immerhin scheint uns ein solches Anforderungsprofil einigermaßen mehrheitsfähig zu sein. Können wir uns auf diese Definition einigen, dann tritt die Frage der beruflichen Grundausbildung in den Hintergrund.

\section{Psychotherapie für wen nicht?}

Bleibt zum Schluss die schwierige Frage, ob es Krankheiten, Störungen oder Problemfelder gibt, die explizit nicht psychotherapeutisch behandelt werden sollten. Nach Senf und Broda bezweckt Psychotherapie die Behandlung oder Prävention psychisch bedingter oder mitbedingter Krankheiten, Störungen und Beschwerden [21]. Wenn wir diese Definition akzeptieren, sollten wir keine Problemfelder ausschließen, solange wir uns im Bereich der psychischen Störungen bewegen, $d$. h. solange beim Patienten eine psychiatrische Erkrankung diagnostiziert worden ist. Auch für leichtere und weniger lang dauernde Störungen wie z. B. die Anpassungsstörungen, bei welchen der empirische Wirksamkeitsnachweis weniger robust ist als beispielsweise bei den Depressionen oder Angststörungen, würden wir eine klare Indikation für Psychotherapie bejahen. Und auch Menschen mit psychi- 
schen Erkrankungen, die primär medikamentös behandelt werden, sollten zusätzlich Psychotherapie erhalten, um individuelle Therapieziele zu erreichen und die Rückfallgefahr zu vermindern. Die Medizin ist keine exakte Wissenschaft. Auch und vor allem in der somatischen Medizin gibt es viele Krankheitsbilder, für die es keine evidenzbasierten Therapierichtlinien gibt. Dennoch muss der Arzt eine Behandlung in die Wege leiten, und in aller Regel wird er von den Krankenkassen auch dafür entschädigt, wenn er seine therapeutische Entscheidung vernünftig begründen kann.

Psychotherapie ist eine Behandlungsmethode für psychische Erkrankungen. Psychisch gesunde Menschen sollten nicht psychotherapeutisch behandelt werden, jedenfalls nicht auf Kosten des öffentlichen Gesundheitswesens (mit der gleichen Überlegung würden wir uns auch gegen „Chirurgie für alle“ oder „Antibiotika für alle“ aussprechen). Zumindest für das Feld der Psychotraumatologie gibt es für diese Haltung auch eine entsprechende wissenschaftliche Grundlage: Es hat sich gezeigt, dass sog. „early interventions“, d. h. eine oder mehrere psychologische Interventionen innerhalb von 3 Monaten nach einem traumatischen Ereignis, nur dann einen Beitrag zur Prävention chronischer posttraumatischer Belastungsstörungen leisten, wenn die Betroffenen unter klinisch relevanten psychopathologischen Symptomen leiden; noch deutlicher wird die Wirkung solcher früher Interventionen bei Vorliegen einer akuten Belastungsstörung oder einer akuten PTSD $[17,18]$.

Wir wollen der Psychotherapie keineswegs „Tür und Tor öffnen“. Psychotherapie wird ohnehin aufgrund der geschilderten Situation weltweit auf absehbare Zeit nur einem kleinen Segment privilegierter Patientinnen und Patienten vorbehalten bleiben. Auch wenn Psychotherapie im Vergleich zu anderen medizinischen Leistungen sehr günstig ist und das Gesundheitswesen insgesamt nur im Prozentbereich finanziell belastet, werden ökonomische Überlegungen den $\mathrm{Zu}$ gang zu Psychotherapie als Krankenkassenleistung in der Zukunft voraussichtlich zunehmend limitieren. Und schließlich: Wie jede wirksame Heilmethode hat auch die Psychotherapie ihre Risiken und Nebenwirkungen [10]. Psychotherapiepatienten können von ihrem Therapeuten abhängig werden. Sie können emotional ausgenützt und im Extremfall sexuell missbraucht werden. Schon nur eine misslingende therapeutische Beziehung kann für vulnerable Patienten eine große Enttäuschung sein. Wenn der Therapieerfolg trotz großem Aufwand und allseitigem Bemühen ausbleibt, stellt dies nicht selten für die Patienten einen schweren Rückschlag dar und wird als persönliches Versagen interpretiert. Die Indikation zur Psychotherapie soll deshalb immer sorgsam, mit Augenmaß, unter Berücksichtigung der verfügbaren wissenschaftlichen Evidenz und klinischer Erfahrungswerte gestellt werden.

\section{Fazit für die Praxis}

- Menschen ohne psychische Erkrankungen sollten nicht psychotherapeutisch behandelt werden, jedenfalls nicht auf Kosten des öffentlichen Gesundheitswesens, zumal es hierfür keine Wirksamkeitsnachweise gibt.

- Heute werden viele Patienten psychotherapeutisch behandelt, die eher zu den leicht kranken Menschen gezählt werden. Psychotherapie sollte zukünftig vermehrt den sehr schwer kranken Menschen angeboten werden.

- Psychotherapie ist eine intellektuell und emotional anspruchsvolle Tätigkeit. Psychotherapeuten sollten dementsprechend akademisch ausgebildet, beziehungsfähig und klinisch versiert sein. Idealerweise verfügen sie über eine gewisse persönliche Reife und Selbstreflexionsfähigkeit.

- Patienten sollen nicht irgendeine, sondern eine möglichst wirksame Psychotherapie erhalten. Die Dissemination empirisch abgesicherter Therapieverfahren muss verbessert werden. Hierzu werden auch sog.,,telemental health approaches" beitragen, in Ergänzung zur klassischen „Face-toface"-Psychotherapie.

\section{Korrespondenzadresse}

Prof. Dr. U. Schnyder

Klinik für Psychiatrie und Psychotherapie,

Universitätsspital Zürich

Culmannstr. 8, 8091 Zürich

Schweiz

ulrich.schnyder@access.uzh.ch

\section{Einhaltung ethischer Richtlinien}

Interessenkonflikt. U. Schnyder, R.M. McShine, J. Kurmann und M. Rufer geben an, dass kein Interessenkonflikt besteht.

Dieser Beitrag beinhaltet keine Studien an Menschen oder Tieren.

\section{Literatur}

1. Chambless DL, Hollon SD (1998) Defining empirically supported therapies. J Consult Clin Psychol 66:7-18

2. DGPPN-Mitteilungen (2010) Bericht zum achten Hauptstadtsymposium. Ärztliche Psychotherapie: Warum, wie und für wen? Nervenarzt 81:768-770

3. Grawe K (2004) Neuropsychotherapie. Hogrefe, Göttingen

4. Grawe K, Donati R, Bernauer F (Hrsg) (1994) Psychotherapie im Wandel. Hogrefe, Göttingen

5. Haug TT, Blomhoff S, Hellstrøm K et al (2003) Exposure therapy and sertraline in social phobia: 1-year follow-up of a randomised controlled trial. Br J Psychiatry 182:312-318

6. Howard KI, Orlinsky DE, Lueger RJ (1994) Clinically relevant outcome research in individual psychotherapy. New models guide the researcher and clinician. Br J Psychiatry 165:4-8

7. Huhn M, Tardy M, Spinelli LM et al (2014) Efficacy of pharmacotherapy and psychotherapy for adult psychiatric disorders: a systematic overview of meta-analyses. JAMA Psychiatry 71:706-715

8. Kandel ER (1998) A new intellectual framework for psychiatry. Am J Psychiatry 155:457-469

9. Knaevelsrud C, Maercker A (2006) Does the quality of the working alliance predict treatment outcome in online psychotherapy for traumatized patients? J Med Internet Res 8(4):e31

10. Leitner A, Märtens M, Koschier A et al (2013) Patients' perceptions of risky developments during psychotherapy. J Contemporary Psychother 43:95-105

11. Lutz W (Hrsg) (2003) Die Wiederentdeckung des Individuums in der Psychotherapieforschung. Ein Beitrag zur patientenorientierten Psychotherapieforschung und Qualitätssicherung. dgvt-Verlag, Tübingen

12. Lutz W (2003) Efficacy, effectiveness, and expected treatment response in psychotherapy. J Clin Psychol 59:745-750

13. Lutz W, Grawe K (2001) Was ist "Evidenz" in einer Evidence Based Psychotherapy? Integrative Therapie 27:11-28

14. Margraf J (Hrsg) (2009) Kosten und Nutzen der Psychotherapie. Eine kritische Literaturauswertung. Springer, Heidelberg 
15. Marks IM, Kenwright M, Mcdonough M et al (2004) Saving clinicians' time by delegating routine aspects of therapy to a computer: a randomized controlled trial in phobia/panic disorder. Psychol Med 34:9-18

16. Perednia DA, Allen A (1995) Telemedicine technology and clinical applications. J Am Med Assoc 273:483-488

17. Roberts NP, Kitchiner NJ, Kenardy J et al (2009) Systematic review and meta-analysis of multiple-session early interventions following traumatic events. Am J Psychiatry 166:293-301

18. Rose S, Bisson J, Churchill R et al (2009) Psychological debriefing for preventing post traumatic stress disorder (PTSD). Cochrane Collaboration. JohnWiley \& Sons

19. Rufer M, Gerke W, Schnyder U (2012) Wie gewinnen wir unseren Nachwuchs? Der Züricher Studienschwerpunkt Psychiatrie und das Weiterbildungskonzept Ärztliche Psychotherapie. Psychiatrie 2:105-110

20. Schnyder U (2009) Future perspectives in psychotherapy. Eur Arch Psychiatry Clin Neurosci 259:S123-S128

21. Senf W, Broda M (Hrsg) (2011) Praxis der Psychotherapie - ein integratives Lehrbuch. Thieme, Stuttgart

22. So $M$, Yamaguchi S, Hashimoto $S$ et al (2013) Is computerised CBT really helpful for adult depression? A meta-analytic re-evaluation of CCBT for adult depression in terms of clinical implementation and methodological validity. BMC Psychiatry 13:113

23. Tseng W-S, Streltzer J (Hrsg) (2001) Culture and psychotherapy. A guide to clinical practice. American Psychiatric Press, Washington DC

\section{springermedizin.de - komfortabel recherchieren in der e.Bibliothek}

Ohne Umwege zur gewünschten Information zu gelangen - springermedizin.de macht's möglich. Verzichten Sie auf überquellende Stehsammler und unübersichtliche Papierstapel, und nutzen Sie statt dessen die digitale e.Bibliothek von Springer Medizin.

Suchen Sie Beiträge in einer bestimmten Fachzeitschrift Ihres Fachgebiets? Oder möchten Sie englischsprachige Journals für eine interdisziplinäre Recherche nutzen? Interessieren Sie sich für Übersichtsbeiträge oder aktuelle wissenschaftliche Studien?

Die e.Bibliothek wird all diesen Anforderungen gerecht: Sie umfasst über 600 deutschsprachige und internationale Fachzeitschriften aus allen Bereichen der Medizin inklusive der medizinischen Inhalte von SpringerLink.

Die e.Bibliothek beinhaltet auch „Online First"-Beiträge, also hoch aktuelle Beiträge, die bereits vor Erscheinen einer gedruckten Ausgabe in elektronischer Form publiziert wurden.

\section{Komfortable und schnelle}

Recherche

- Mit der Volltextsuche von springermedizin.de durchsuchen Sie den gesamten Inhalt der e.Bibliothek und gelangen direkt zu den Inhalten, die für Sie relevant sind.

- AufWunsch können Sie die Suchergebnisse eingrenzen und beispielsweise gezielt in einzelnen Zeitschriften, nach Themen und sogar nach Autoren suchen.

- Wenn Sie einen englischen Suchbegriff eingeben, erhalten Sie zudem Treffer aus den englischsprachigen wissenschaftlichen Zeitschriften von SpringerLink.
Mit der Suchfunktion gelangen Sie auch zu Inhalten aus allen anderen Bereichen von springermedizin.de von zertifizierten Fortbildungskursen der e.Akademie, aktuellen Kongressberichten bis hin zu aktuellen Themendossiers, Videos und Nachrichten aus der Gesundheits- und Berufspolitk.

\section{Ihre persönliche Merkliste}

Finden Sie einen Beitrag besonders interessant oder möchten Sie inn für die spätere Lektüre vormerken? Auf springermedizin.de können Sie ganz einfach Ihre persönliche Merkliste anlegen: ein Klick auf das Symbol "merken“ am Beitragsende genügt und die Beiträge erscheinen unter "Meine Merkliste".

Alle Beiträge sind als PDF-Datei im Layout der gedruckten Ausgabe sowie als HTML-Version verfügbar. In der HTMLVersion können Sie die Vorteile der verlinkten Literatur nutzen und direkt zu den zitierten Quellen gelangen.

e.Med - der Zugang zu allen digitalen Inhalten von Springer Medizin

Zugang zu allen Inhalten der e.Bibliothek bekommen Sie mit e.Med. Lernen Sie die Vorzüge dieses umfassenden Angebots kennen und testen Sie e.Med 30 Tage lang kostenlos und unverbindlich unter

\section{www.springermedizin.de/eMed}

Eine erfolgreiche Recherche wünscht Ihnen Ihr Redaktionsteam Fachzeitschriften von Springer Medizin 